\title{
Impact of Population Stratification on Family-Based Association in an Admixed Population
}

\author{
T. B. Mersha, ${ }^{1,2}$ L. Ding, ${ }^{1,2}$ H. He, ${ }^{1}$ E. S. Alexander, ${ }^{1,3}$ X. Zhang, ${ }^{1}$ B. G. Kurowski, ${ }^{1,2}$ \\ V. Pilipenko, ${ }^{1}$ L. Kottyan, ${ }^{1,2}$ L. J. Martin, ${ }^{1,2}$ and D. W. Fardo ${ }^{4}$ \\ ${ }^{1}$ Divisions of Asthma Research, Biostatistics and Epidemiology, Human Genetics, and Physical Medicine and Rehabilitation and \\ Center for Autoimmune Genomics and Etiology, Cincinnati Children's Hospital Medical Center, Cincinnati, OH 45229, USA \\ ${ }^{2}$ Department of Pediatrics, University of Cincinnati College of Medicine, Cincinnati, OH 45267, USA \\ ${ }^{3}$ Health Services Administration, Xavier University, Cincinnati, OH 45207, USA \\ ${ }^{4}$ Department of Biostatistics, University of Kentucky, Lexington, KY 40536, USA
}

Correspondence should be addressed to D. W. Fardo; david.fardo@uky.edu

Received 10 December 2014; Accepted 7 April 2015

Academic Editor: Graziano Pesole

Copyright (C) 2015 T. B. Mersha et al. This is an open access article distributed under the Creative Commons Attribution License, which permits unrestricted use, distribution, and reproduction in any medium, provided the original work is properly cited.

Population substructure is a well-known confounder in population-based case-control genetic studies, but its impact in familybased studies is unclear. We performed population substructure analysis using extended families of admixed population to evaluate power and Type I error in an association study framework. Our analysis shows that power was improved by $1.5 \%$ after principal components adjustment. Type I error was also reduced by $2.2 \%$ after adjusting for family substratification. The presence of population substructure was underscored by discriminant analysis, in which over $92 \%$ of individuals were correctly assigned to their actual family using only 100 principal components. This study demonstrates the importance of adjusting for population substructure in family-based studies of admixed populations.

\section{Introduction}

Complex diseases result from the interplay of multiple genetic and environmental factors. To study the genetic basis of complex diseases, two broad types of study designs-populationbased and family-based-are often used. Population-based designs sample individuals who are unrelated, as in casecontrol studies. Family-based designs use related individuals, often sampled through a proband. Case-control design is based on allele or genotype frequencies comparison of unrelated affected and unaffected individuals in the population [1]. An allele in a gene is said to be associated with a trait if it occurs at a significantly different frequency in the affected individuals compared to the control group (i.e., when the null hypothesis of equal allele frequency across groups is false). Family-based designs use groups of trios, nuclear families, or extended families. Family studies can address whether a disease aggregates in families [2]. Such studies typically examine correlations between traits and deviations from the allele transmissions expected assuming Mendelian inheritance. Although case-control designs have practical advantages over family-based designs in sample recruitment and collecting DNA from unrelated cases and controls, family-based association studies have received much attention in the literature because of their robustness to population stratification and higher power to detect very rare variants with small effects compared to case-control studies. Population stratification is present when the population includes several subpopulations, and the allele frequency of interest differs in each subpopulation due to systematic differences in ancestry rather than association of variants with disease $[3,4]$. Although most population stratifications occur when there are multiple races or ethnicities in case-control study design, significant population stratification can be identified even within an apparently homogeneous North American population of European ancestry [5]. Recent study found that individuals, who are identified as white, have about $3.5 \%$ nonEuropean ancestry [6]. 
By contrast, family-based association analyses assume that individual family members come from a common genetic background, and families tend to be more homogeneous regarding exposure to environmental factors that may be associated with the disease etiology. Hence, the analysis of phenotypes among family members is moderately controlled for both genetic background and environmental exposures. Because family members share a predictable proportion of their genes identical by descent, the background genetic variation is somewhat controlled as a function of the degree of relationship (or kinship coefficient) and modeled as a polygenic component [7]. This strong assumption of population homogeneity, however, is often untenable, and many association studies include samples from structured family members or admixed individuals. The world is becoming highly multiethnic, and intermarriage between different groups is becoming more and more common [8]. In the United States, the two major admixed populations are the African and Latino Americans. Populations like African Americans and Latino Americans were formed within the past 400 years (i.e., within approximately 10 generations) [9]. Therefore, the standard approach of selecting all individuals from the same population/ethnic group and geographic area is not always possible. In an extended family, many of the loci may be unique or may greatly vary in frequency within and between family members. False-positive associations (Type I errors) occur when the frequencies of genetic markers and the disease of interest vary across different subpopulation groups $[10,11]$. Data from the San Antonio Family Study (SAFS) provided through Genetic Analysis Workshop 18 [12] are a classic example in which related individuals were recruited from admixed Mexican American families. In such situations, failure to appropriately account for pedigree structure in family-based study can lead to spurious associations. Here, we consider accounting for family structure in admixed ancestry. Using an admixed population from SAFS, our study found that there is power to be gained by accounting for family structure in family-based association studies of an admixed population.

\section{Materials and Methods}

The Genetic Analysis Workshop 18 (GAW18) data consists of whole-genome sequences from extended pedigrees [13]. The GAW18 dataset was created to provide a platform for developing and evaluating relevant statistical methods [12]. We analyzed whole-genome sequencing data from chromosome 3 and performed data cleaning to select high quality SNPs and avoid Mendelian errors. Twenty large pedigrees from SAFS ranging from 22 to 86 members in size and recruited from a Mexican American population generated 1,215,399 SNPs genotyped on 959 individuals. Mexican Americans are an admixed population with a contribution of European, Amerindian, and African ancestries [14]. We performed principal components analysis (PCA) using 10,000 randomly selected common variants (minor allele frequency (MAF) $>0.1)$ to investigate the family substratification. The first two principal components (PCs) revealed that families were clustered together and accounted for $2.19 \%$ and $1.47 \%$ of the

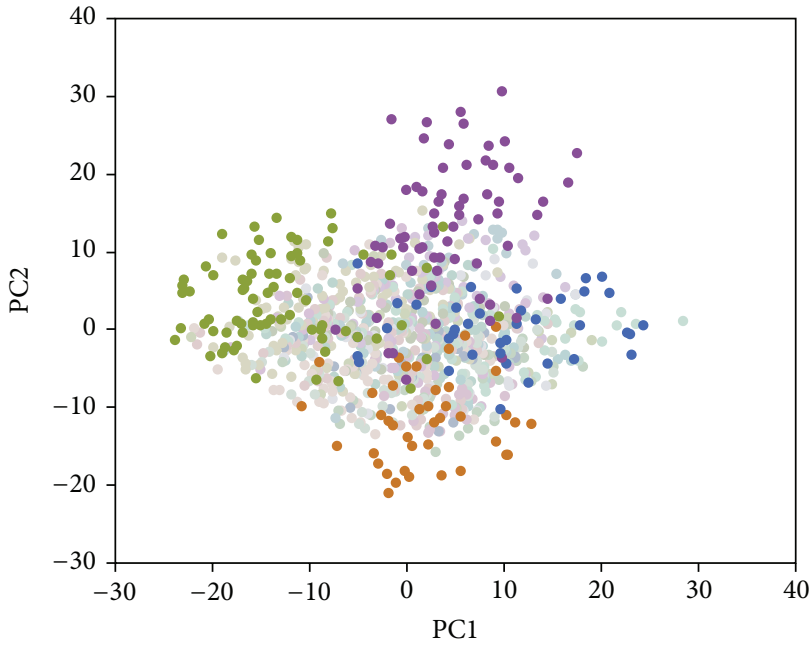

FIgURE 1: The first two principal components (PCs) using 10,000 randomly selected variants. The 20 large pedigrees from SAFS range from 22 to 86 family members.

total genetic variation, respectively (Figure 1). Two families (families 3 and 14 in Table 1) showed a marked difference in the first PC and two additional families (families 5 and 15) showed clear distinction in the second PC. These two pairs of families were used to select informative markers and capture the variation in this multigenerational structure pedigree. Analysis showed that 95 and 291 PCs were needed to explain $50 \%$ and $80 \%$ of the total variation, respectively. Our goal was to identify divergent family members within the extended pedigree and create a homogeneous family structure.

We calculated the MAF in each of these 4 families for the 1,215,399 SNPs. The absolute allele frequency difference (delta) was used to measure marker informativeness between the two paired divergent families mentioned above. Marker informativeness for ancestry was ascertained through the absolute value of the difference in the frequency of the minor allele from the $m$ th SNP observed for the 2 populations [15]. If we let $p_{m 1}$ represent the frequency of a reference allele in the first population and $p_{m 2}$ the frequency of the same allele in the second population, then the delta value is given by $\delta=$ $\left|p_{m 1}-p_{m 2}\right|$. Markers with different frequency distributions among populations can be used to adjust for population stratification among admixed populations. Using a cutoff value of 0.6 for delta $(\delta>0.6$, a cutoff that has been suggested as highly informative for discriminating between European and African ancestry) [16], 218 ancestry informative markers (AIMs) were selected from 1,215,399 SNPs using families 5 and 15; similarly, 404 variants were selected as AIMs for families 3 and 14. Given the vast amount of sequence data, data mining can be a fast and cost-effective approach for investigating the number of SNPs that are required to discriminate between populations. AncestrySNPminer is a web-based bioinformatics tool specifically designed to retrieve informative markers between populations with different allele frequency. The tool includes an automated and simple "scripting at the click of a button" functionality 
TABLE 1: Number of correctly classified family members per family using different numbers of PCs. These are boldfaced when stably reaching the total family size.

\begin{tabular}{|c|c|c|c|c|c|c|c|c|c|c|c|c|}
\hline \multirow{2}{*}{ Family ID } & \multirow{2}{*}{ Number of family members } & \multicolumn{11}{|c|}{ Number of PCs } \\
\hline & & 1 & 20 & 40 & 60 & 80 & 100 & 120 & 140 & 160 & 180 & 200 \\
\hline 2 & 86 & 10 & 47 & 67 & 72 & 75 & 78 & 81 & 84 & 86 & 86 & 86 \\
\hline 3 & 77 & 77 & 73 & 76 & 75 & 76 & 76 & 77 & 77 & 77 & 77 & 77 \\
\hline 4 & 64 & 0 & 42 & 49 & 54 & 57 & 58 & 61 & 62 & 62 & 62 & 63 \\
\hline 5 & 68 & 0 & 54 & 65 & 65 & 66 & 66 & 68 & 68 & 68 & 68 & 68 \\
\hline 6 & 64 & 28 & 33 & 48 & 50 & 54 & 56 & 57 & 58 & 61 & 62 & 62 \\
\hline 7 & 36 & 0 & 14 & 19 & 25 & 26 & 30 & 33 & 33 & 33 & 33 & 33 \\
\hline 8 & 68 & 0 & 37 & 56 & 60 & 65 & 66 & 67 & 67 & 68 & 68 & 68 \\
\hline 9 & 33 & 0 & 10 & 21 & 27 & 31 & 30 & 29 & 31 & 31 & 31 & 31 \\
\hline 10 & 64 & 0 & 38 & 50 & 55 & 59 & 61 & 61 & 63 & 63 & 63 & 64 \\
\hline 11 & 35 & 0 & 22 & 25 & 29 & 30 & 31 & 33 & 33 & 34 & 35 & 34 \\
\hline 14 & 40 & 0 & 25 & 32 & 33 & 38 & 39 & 40 & 39 & 39 & 40 & 40 \\
\hline 15 & 41 & 0 & 28 & 33 & 37 & 38 & 38 & 39 & 40 & 40 & 41 & 41 \\
\hline 16 & 48 & 0 & 31 & 40 & 41 & 43 & 42 & 45 & 45 & 47 & 46 & 47 \\
\hline 17 & 42 & 5 & 31 & 33 & 38 & 37 & 38 & 39 & 40 & 42 & 41 & 42 \\
\hline 20 & 36 & 0 & 15 & 24 & 32 & 31 & 32 & 32 & 32 & 36 & 35 & 35 \\
\hline 21 & 35 & 0 & 20 & 21 & 29 & 32 & 33 & 33 & 33 & 33 & 34 & 35 \\
\hline 23 & 32 & 0 & 15 & 25 & 27 & 26 & 29 & 28 & 31 & 31 & 30 & 31 \\
\hline 25 & 33 & 0 & 21 & 25 & 26 & 27 & 30 & 30 & 30 & 30 & 30 & 31 \\
\hline 27 & 35 & 0 & 18 & 26 & 27 & 29 & 33 & 34 & 34 & 33 & 34 & 35 \\
\hline 47 & 22 & 0 & 19 & 17 & 19 & 19 & 19 & 19 & 21 & 21 & 21 & 21 \\
\hline
\end{tabular}

that enables researchers to perform user-friendly querying and filtering of databases across various publicly available or investigator uploaded datasets through a single web interface. The results can be downloaded or viewed in the browser where users can interactively explore linkage disequilibrium patterns and allele frequency differences among variants (https://research.cchmc.org/mershalab/) [17]. To account for population stratification, we performed PCA using all of the selected 622 AIMs to infer continuous axes of genetic variation. To assess the number of PCs needed for accurate individual family member assignment, we applied linear discriminant analysis. All analyses were run in $\mathrm{R}$ version 3.1.3 (http://cran.r-project.org/) [18] and packages MASS [19] (lda function) and nlme [20] (lme function) were used to run linear discriminant analysis and linear mixed modeling, respectively.

\section{Results and Discussion}

The first two principal components using the 622 AIMs accounted for $16.4 \%$ and $12.0 \%$ of the total variation, respectively, revealing an increased separation of the 20 families (Figure 2). The analysis required 8 and 35 PCs to explain 50\% and $80 \%$ of the total variation, respectively. To predict family membership, we applied linear discriminant analysis on the principal components obtained from the 622 AIMs. Table 1 describes the number of family members that were correctly allocated to each corresponding family using different numbers of PCs. Notably, the number of correctly classified family members for family 3 is 77 out of 77 when using a

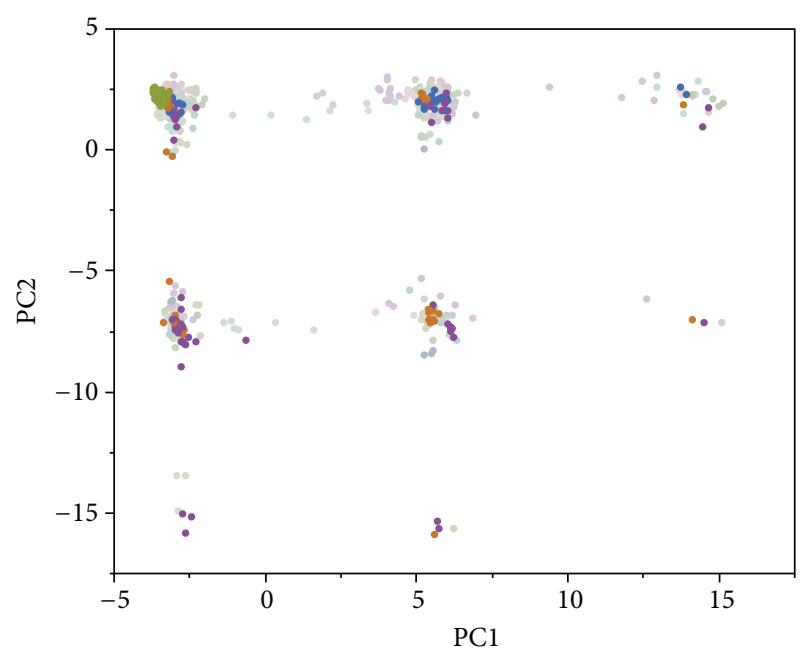

FIGURE 2: The first two principal components (PCs) using 622 AIMs. The 20 large pedigrees from SAFS range from 22 to 86 family members.

single PC. Adding additional PCs can introduce noise but the classification accuracy levels as more PCs are utilized. Using ancestry informative markers, the classification accuracy is $12.5 \%$ for 1 PC and $92.8 \%$ for 100 PCs. In order to investigate the impact of population stratification on family-based association in an admixed population, a linear mixed model was employed to test for each SNP's association with systolic blood pressure. Blood pressure medication use, gender, age, 
TABLE 2: Improved power and reduced Type I error.

\begin{tabular}{lcc}
\hline & Without PC & With PC \\
\hline Power & 0.142 & 0.157 \\
Type I error & 0.120 & 0.098 \\
\hline
\end{tabular}

and gender-by-age interaction were included as fixed effects in the model. We also include family as a random effect. Here, we do not explicitly model familial relationships in the random effect variance as in GRAMMAR [21] and famSKAT [22]. Based on the scree plot, we used the first six principal components (derived from 622 AIMs) as covariates to adjust for population stratification. 134 causal SNPs as simulated in GAW18 were used to assess power [13]. A null trait was used to access the Type I error based on randomly selected 10,000 SNPs. Table 2 shows that the power was slightly improved by $1.5 \%$ after PC adjustment. Table 2 also suggests that the Type I error was also reduced by $2.2 \%$ after we adjusted for the family substratification.

\section{Conclusions}

Accounting for population structure is more challenging when family structure or cryptic relatedness is present in an admixed population created from multiple ancestral populations. In this study, we performed population substructure analysis using extended families from admixed Mexican American population and evaluated power and Type I error in family-based association framework. Our analysis shows that power was improved by $1.5 \%$ after principal components adjustment. Type I error was also reduced by $2.2 \%$ after adjusting for family substratification. Our findings demonstrate that the traditional wisdom of family-based association studies being guarded against spurious association due to population stratification only holds when the background genetic variation is properly accounted for. The broad assumption in family-based study design is that individuals come from common genetic background among the family members. Unless this background is explicitly controlled for as in family-based association tests, or FBATs [23], robustness to population substructure may not be guaranteed. Moreover, families tend to be more homogeneous regarding exposure to environmental factors and characterized by "environmental homogeneity" that may be associated with the disease etiology. However, in an extended family of admixed population, there is also multigenerational structure created within-family and between-families, leading to unusual allele frequency differences among subgroups. Thus, it is critical to correct for stratification in family-based samples exhibiting admixture. Using an admixed population from SAFS, we accounted for relatedness and structure within admixed populations by using individual-specific allele frequencies at SNPs that are calculated on the basis of between-family variance derived from 622 SNPs serving as AIMs. Failure to appropriately account for pedigree structure can lead to spurious association (i.e., false-positive findings). Modelling family structure is a necessity in studies with family-based sample ascertainment, and there is increasing evidence that cryptic relatedness may occur in a wide range of data sets as shown in our study. Our approach offers potential solution for dealing with family structure in family-based studies. Future studies using mixed models that incorporate the full covariance structure across related individuals and model them as a polygenic component will be essential. In addition, families of Mexican American admixed genetic structure present unique opportunities to explore the genetic etiology of complex disease using admixture mapping. Mapping susceptibility genes in an admixed population using admixture mapping involves screening the genome of individuals of mixed ancestry, who have the disease, for chromosomal regions that have a greater percentage of alleles from the parental population with the higher disease risk [24]. GWAS has successfully identified common SNPs associated with many diseases. Family-based designs which include families enriched with rare genetic susceptibilities can have more power to detect genetic effects than unrelated samples given an equivalent number of sampling units [25]. Future studies integrating family-based linkage, association, and admixture mapping could help to efficiently map genomic regions associated with disease risk [26].

\section{Disclaimer}

The funders had no role in study design, data collection and analysis, decision to publish, or preparation of the paper.

\section{Conflict of Interests}

The authors declare that there is no conflict of interests regarding the publication of this paper.

\section{Acknowledgments}

This work was partially supported by National Institutes of Health Grants K01HL103165, K23HD074683, and K25AG043546. The authors would like to thank the organizers of GAW18 for providing access to the dataset. The GAW18 whole-genome sequence data were provided by the T2DGENES Consortium, which is supported by NIH Grants U01 DK085524, U01 DK085584, U01 DK085501, U01 DK085526, and U01 DK085545. The other genetic and phenotypic data for GAW18 were provided by the San Antonio Family Heart Study and San Antonio Family Diabetes/Gallbladder Study, which are supported by NIH Grants P01 HL045222, R01 DK047482, and R01 DK053889. The Genetic Analysis Workshop is supported by NIH Grant R01 GM031575.

\section{References}

[1] N. Risch and K. Merikangas, "The future of genetic studies of complex human diseases," Science, vol. 273, no. 5281, pp. 15161517, 1996.

[2] J. W. Smoller and C. T. Finn, "Family, twin, and adoption studies of bipolar disorder," American Journal of Medical GeneticsSeminars in Medical Genetics, vol. 123, no. 1, pp. 48-58, 2003.

[3] W. J. Ewens and R. S. Spielman, "The transmission/disequilibrium test: history, subdivision, and admixture," The American Journal of Human Genetics, vol. 57, no. 2, pp. 455-464, 1995. 
[4] J. K. Pritchard and P. Donnelly, "Case-control studies of association in structured or admixed populations," Theoretical Population Biology, vol. 60, no. 3, pp. 227-237, 2001.

[5] C. D. Campbell, E. L. Ogburn, K. L. Lunetta et al., "Demonstrating stratification in a European American population," Nature Genetics, vol. 37, no. 8, pp. 868-872, 2005.

[6] K. Bryc, E. Y. Durand, J. M. Macpherson, D. Reich, and J. L. Mountain, "The genetic ancestry of African Americans, Latinos, and European Americans across the United States," The American Journal of Human Genetics, vol. 96, no. 1, pp. 37-53, 2015.

[7] I. B. Borecki and M. A. Province, "Genetic and genomic discovery using family studies," Circulation, vol. 118, no. 10, pp. 1057-1063, 2008.

[8] G. Hellenthal, G. B. J. Busby, G. Band et al., "A genetic atlas of human admixture history," Science, vol. 343 , no. 6172, pp. 747751, 2014.

[9] M. W. Smith, N. Patterson, J. A. Lautenberger et al., "A highdensity admixture map for disease gene discovery in African Americans," The American Journal of Human Genetics, vol. 74, no. 5, pp. 1001-1013, 2004.

[10] M. L. Freedman, D. Reich, K. L. Penney et al., "Assessing the impact of population stratification on genetic association studies," Nature Genetics, vol. 36, no. 4, pp. 388-393, 2004.

[11] J. Marchini, L. R. Cardon, M. S. Phillips, and P. Donnelly, "The effects of human population structure on large genetic association studies," Nature Genetics, vol. 36, no. 5, pp. 512-517, 2004.

[12] H. Bickeböller, J. N. Bailey, J. Beyene et al., "Genetic analysis workshop 18: methods and strategies for analyzing human sequence and phenotype data in members of extended pedigrees," BMC Proceedings, vol. 8, supplement 1, article S1, 2014.

[13] L. Almasy, T. D. Dyer, J. M. Peralta et al., "Data for Genetic Analysis Workshop 18: human whole genome sequence, blood pressure, and simulated phenotypes in extended pedigrees," BMC Proceedings, vol. 8, supplement 1, article s2, 2014.

[14] H.-Q. Qu, Q. Li, Y. Lu, S. P. Fisher-Hoch, and J. B. McCormick, "Translational genomic medicine: common metabolic traits and ancestral components of Mexican Americans," Journal of Medical Genetics, vol. 49, no. 8, pp. 544-545, 2012.

[15] L. Ding, H. Wiener, T. Abebe et al., "Comparison of measures of marker informativeness for ancestry and admixture mapping," BMC Genomics, vol. 12, article 622, 2011.

[16] H. E. Collin-Schramm, R. A. Kittles, D. J. Operario et al., "Markers that discriminate between European and African ancestry show limited variation within Africa," Human Genetics, vol. 111, no. 6, pp. 566-569, 2002.

[17] S. Amirisetty, G. K. K. Hershey, and T. M. Baye, "AncestrySNPminer: a bioinformatics tool to retrieve and develop ancestry informative SNP panels," Genomics, vol. 100, no. 1, pp. 57-63, 2012.

[18] R Core Team, R: A Language and Environment for Statistical Computing, R Foundation for Statistical Computing, Vienna, Austria, 2015.

[19] W. N. Venables and B. D. Ripley, Modern Applied Statistics with $S$, Statistics and Computing, Springer, New York, NY, USA, 4th edition, 2002.

[20] J. Pinheiro, D. Bates, S. DebRoy, D. Sarkar, and The R Core Team, nlme: Linear and Nonlinear Mixed Effects Models, 2015.

[21] Y. S. Aulchenko, D.-J. J. de Koning, and C. Haley, "Genomewide rapid association using mixed model and regression: a fast and simple method for genomewide pedigree-based quantitative trait loci association analysis," Genetics, vol. 177, no. 1, pp. 577585, 2007.

[22] H. Chen, J. B. Meigs, and J. Dupuis, "Sequence kernel association test for quantitative traits in family samples," Genetic Epidemiology, vol. 37, no. 2, pp. 196-204, 2013.

[23] S. Horvath, X. Xu, and N. M. Laird, "The family based association test method: strategies for studying general genotypephenotype associations," European Journal of Human Genetics, vol. 9, no. 4, pp. 301-306, 2001.

[24] T. M. Baye and R. A. Wilke, "Mapping genes that predict treatment outcome in admixed populations," Pharmacogenomics Journal, vol. 10, no. 6, pp. 465-477, 2010.

[25] R. Kazma and J. N. Bailey, "Population-based and family-based designs to analyze rare variants in complex diseases," Genetic Epidemiology, vol. 35, supplement 1, pp. S41-S47, 2011.

[26] J. Ott, Y. Kamatani, and M. Lathrop, "Family-based designs for genome-wide association studies," Nature Reviews Genetics, vol. 12, no. 7, pp. 465-474, 2011. 

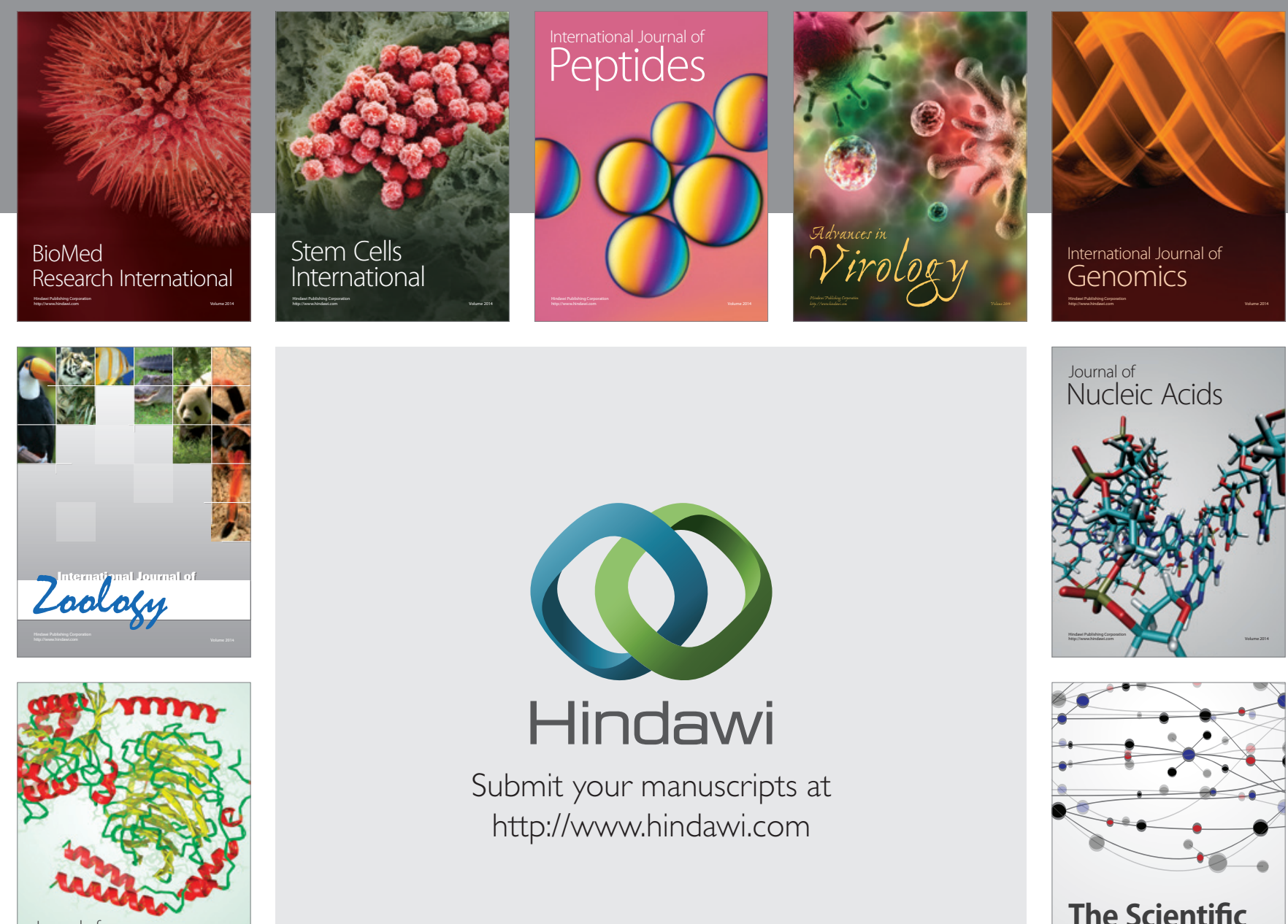

Submit your manuscripts at

http://www.hindawi.com

Journal of
Signal Transduction
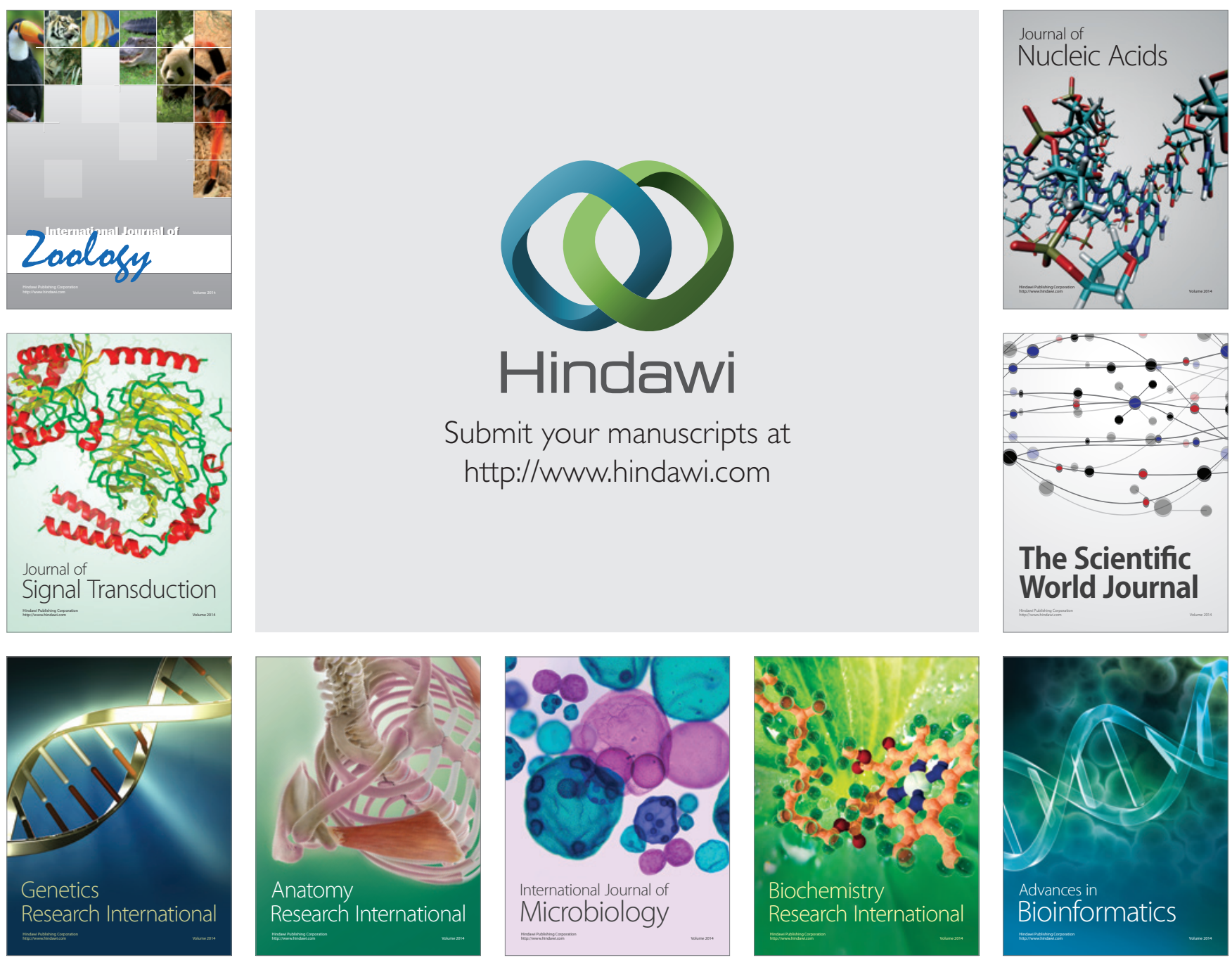

The Scientific World Journal
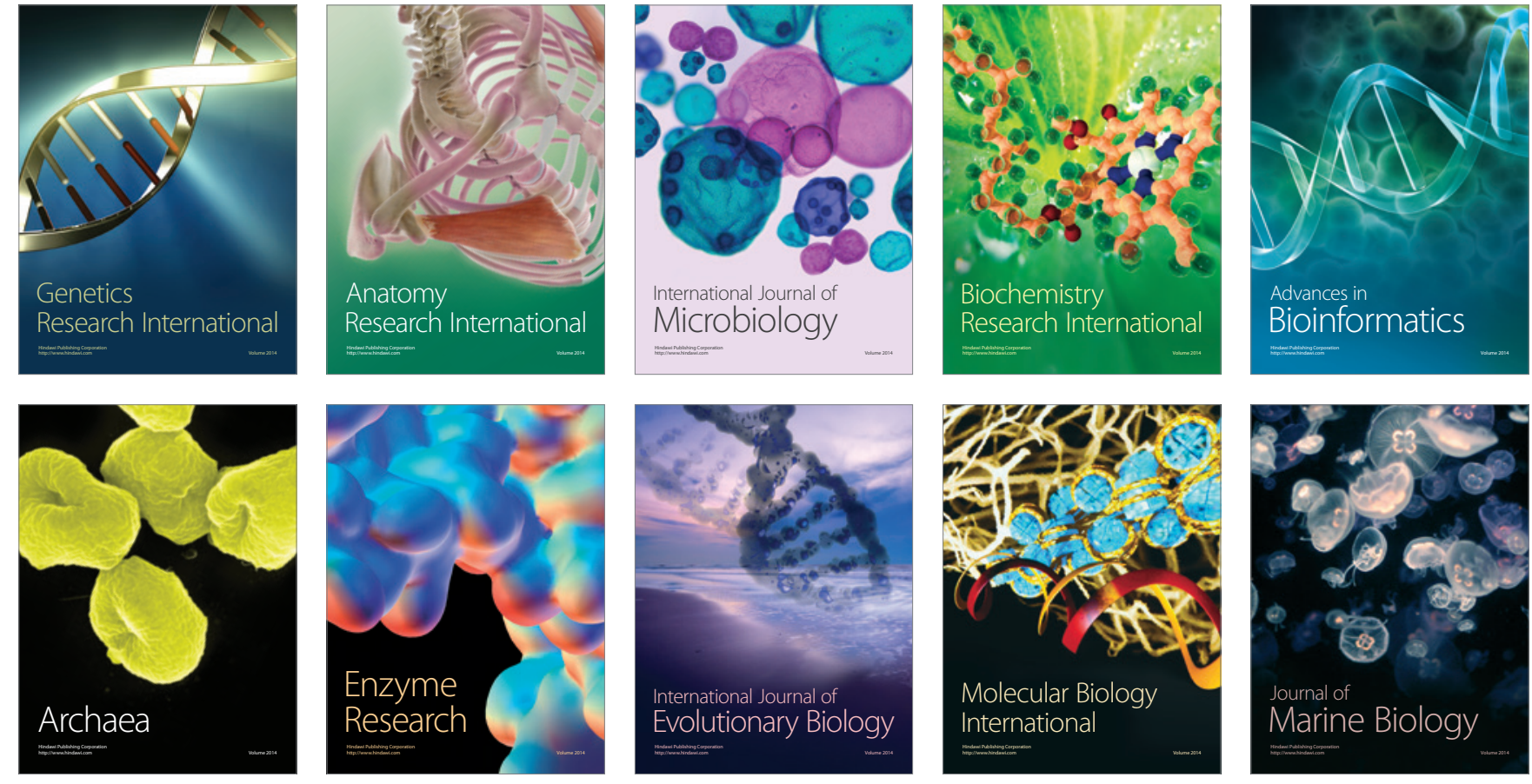